\title{
Thrombomodulin in disseminated intravascular coagulation and other critical conditions - a multi-faceted anticoagulant protein with therapeutic potential
}

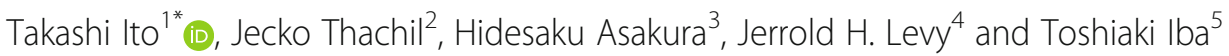

\begin{abstract}
Thrombomodulin plays a vital role in maintaining intravascular patency due to its anticoagulant, antiinflammatory, and cytoprotective properties. However, under pathological conditions such as sepsis and systemic inflammation, endothelial thrombomodulin expression is downregulated and its function impaired. As a result, administering thrombomodulin represents a potential therapeutic modality. Recently, the effect of recombinant thrombomodulin administration in sepsis-induced coagulopathy was evaluated in a randomized controlled study (SCARLET). A 2.6\% 28-day absolute mortality reduction (26.8\% vs. $29.4 \%$ ) was reported in 800 patients studied that was not statistically significant; however, a post hoc analysis revealed a $5.4 \%$ absolute mortality reduction among the patients who fulfilled the entry criterion at baseline. The risk of bleeding did not increase compared to placebo control. Favorable effects of thrombomodulin administration have been reported not only in sepsis-induced coagulopathy but also in disseminated intravascular coagulations with various backgrounds. Interestingly, beneficial effects of recombinant thrombomodulin in respiratory, renal, and cardiovascular diseases might depend on its anti-inflammatory mechanisms. In this review, we summarize the accumulated knowledge of endogenous as well as recombinant thrombomodulin from basic to clinical aspects and suggest future directions for this novel therapeutic agent.
\end{abstract}

Keywords: Recombinant thrombomodulin, Disseminated intravascular coagulation (DIC), Coagulopathy, Randomized controlled trial, Bleeding, Sepsis, Septic shock

\section{Background}

Ever since Esmon et al. first reported the importance of thrombomodulin (TM) as a cofactor for thrombin-catalyzed activation of protein $C$ in the 1980s [1], this important molecule has attracted considerable interest in the field of thrombosis and inflammation. TM was initially considered an important cofactor for the anticoagulant protein $\mathrm{C}$ system, but subsequently, TM was noted to be a critical component of a multimolecular system, integrating major endothelial function that includes antithrombotic, antiinflammatory, and cytoprotective properties [2]. These findings made TM an attractive drug candidate for therapy of diseases where vascular endothelial function was impaired.

\footnotetext{
* Correspondence: takashi@m3.kufm.kagoshima-u.ac.jp

'Department of Systems Biology in Thromboregulation, Kagoshima

University Graduate School of Medical and Dental Sciences, 8-35-1

Sakuragaoka, Kagoshima 890-8544, Japan

Full list of author information is available at the end of the article
}

Accordingly, a recombinant TM (rTM, ART-123, Recomodulin $^{\circ}$ ) was developed and studied for the treatment of disseminated intravascular coagulation (DIC) [3]. Recently, a randomized controlled trial (RCT) that examined the effect of rTM on sepsis-induced coagulopathy was performed [4]. The results of this study and a meta-analysis reported a trend toward favorable outcomes in the treatment groups [5]. In this review, we summarize the physiological role of $\mathrm{TM}$, the potential utility of soluble fragments of TM as a biomarker, and the efficacy of rTM in clinical use.

\section{Physiology of thrombomodulin (TM)}

TM is a type I transmembrane glycoprotein composed of 557 amino acids that is expressed on the luminal surface of endothelial cells where it suppresses thrombus formation by modulating thrombin's procoagulant effects. TM binds reversibly to the anion-binding exosite-I of thrombin,

(c) The Author(s). 2019 Open Access This article is distributed under the terms of the Creative Commons Attribution 4.0 International License (http://creativecommons.org/licenses/by/4.0/), which permits unrestricted use, distribution, and 
interrupting the binding of thrombin to its procoagulant substrates that include fibrinogen, protease-activated receptors, and coagulation factors V and VIII [6]. Conversely, TM provides the binding surface for protein $C$ in such a manner that this anticoagulant zymogen is efficiently activated by thrombin bound to the adjacent portion of TM [6]. As a result, activated protein C (APC), in association with protein $\mathrm{S}$, degrades coagulation factors Va and VIIIa further inhibiting thrombin generation. Endothelial-specific loss of TM results in spontaneous and fatal thrombosis in mice, suggesting that the TM-protein $C$ pathway is critical in preventing intravascular coagulation [7].

Besides the anticoagulant effects, APC has cytoprotective and antiinflammatory effects (Fig. 1) that are mediated by the endothelial protein $C$ receptor (EPCR)-protease-activated receptor 1 (PAR1) system [8]. PAR1 was initially identified as a receptor for thrombin, and thrombin binding induces proinflammatory responses, contributing to the pathogenesis of vascular inflammation. However, PAR1 can be alternatively activated by EPCR-APC, eliciting protective signaling responses in endothelial cells $[8,9]$ that include downregulation of vascular adhesion molecules, stabilization of endothelial barrier function, and inhibition of apoptotic signaling.

Thrombin activatable fibrinolysis inhibitor (TAFI) is another critical substrate for the thrombin-TM complex. Activated TAFI inhibits fibrinolysis by removing lysine residues from the carboxy-terminal region of fibrin, thereby diminishing plasminogen binding to fibrin. TAFI is also important in regulating complement activation [10]. In addition to these actions, TM has a direct inhibitory effect on inflammatory responses that is conferred by the lectin-like domain of TM. Mice lacking this domain are healthy but are vulnerable to neutrophil-mediated tissue damage with inflammatory diseases [11]. There also appears to be multiple mechanisms by which TM's lectinlike domain suppresses inflammatory responses that include suppression of leukocyte adhesion to endothelial cells [11], interference of complement activation [12, 13], inactivation of proinflammatory nuclear proteins, such as high mobility group box 1 (HMGB1) and histones [14], and inactivation of bacterial endotoxin [15]. Interestingly, endotoxin, HMGB1, and histones are recognized by the common pattern recognition receptors, and thus TM binding inhibits the receptor-mediated proinflammatory reactions. Taken together, these findings suggest that the anti-inflammatory effects of TM arise through multi-factorial mechanisms involving both APC-dependent and APC-independent actions [14].

\section{Endogenous soluble TM as a potential biomarker of endothelial injury}

Fragments of the extracellular region of membranebound TM called endogenous soluble TM can be generated by proteolytic cleavage by leukocyte-derived proteases and metalloproteases that are released in sepsis and inflammatory states $[2,16]$. Although these shed fragments can be detected in plasma and urine of healthy individuals, their levels are increased several-fold under certain inflammatory conditions including trauma, DIC, pulmonary thromboembolism, acute respiratory distress syndrome (ARDS), chronic renal failure, or acute hepatic failure $[17,18]$. Soluble TM may independently have some physiological functions as $30-50 \%$ of the anticoagulant activity of cell-associated TM may be preserved in these soluble fragments depending on the fragment length $[19,20]$.

\section{The development of rTM}

The cDNA and amino acid sequence of human TM was first identified by Suzuki et al. in 1987 [21]. The recombinant form (rTM, ART-123, Recomodulin ${ }^{\circ}$ ), which consists of the extracellular portion of TM and lacks the hydrophobic transmembrane and cytoplasmic regions, was subsequently synthesized in Chinese Hamster Ovary cells and was evaluated as a potential anticoagulant. Compared to natural TM, rTM had an approximately equivalent binding affinity for thrombin and cofactor activity for protein $\mathrm{C}$ activation [21]. When dosed clinically, rTM preferentially inhibits thrombin generation through protein $\mathrm{C}$ activation and subsequent factor $\mathrm{Va}$ inactivation, without inhibiting thrombin activity directly [22]. As a result, rTM had minimal effects on clotting times compared to the direct thrombin inhibitor argatroban, indirect thrombin inhibitor heparin, or recombinant APC [22, 23]. Although rTM and APC both molecules share the same anticoagulant mechanism, rTM provides APCdependent anticoagulation only after thrombin is generated. Since plasma APC can be rapidly inactivated by several plasma serine protease inhibitors, rTM is considered to act locally where thrombin is generated [24], a potential reason for the observed low risk of bleeding with rTM therapy compared to APC [25]. Based on the pharmacokinetic data obtained from healthy volunteers and DIC patients, the intravenous infusion of $0.06 \mathrm{mg} / \mathrm{kg}$ rTM once daily was recommended [26, 27]. The half-life of rTM is approximately $20 \mathrm{~h}$, and more than $50 \%$ of the drug is excreted from urine. However, the dose reduction is not necessary even in moderate renal dysfunction [28]. rTM is currently available only in Japan and the cost for 6-day-treatment will be over $\$ 3000$ in US dollars.

\section{The clinical effectiveness of rTM in sepsis}

The theoretical background of the anticoagulant therapy for sepsis-induced coagulation dysfunction has been 


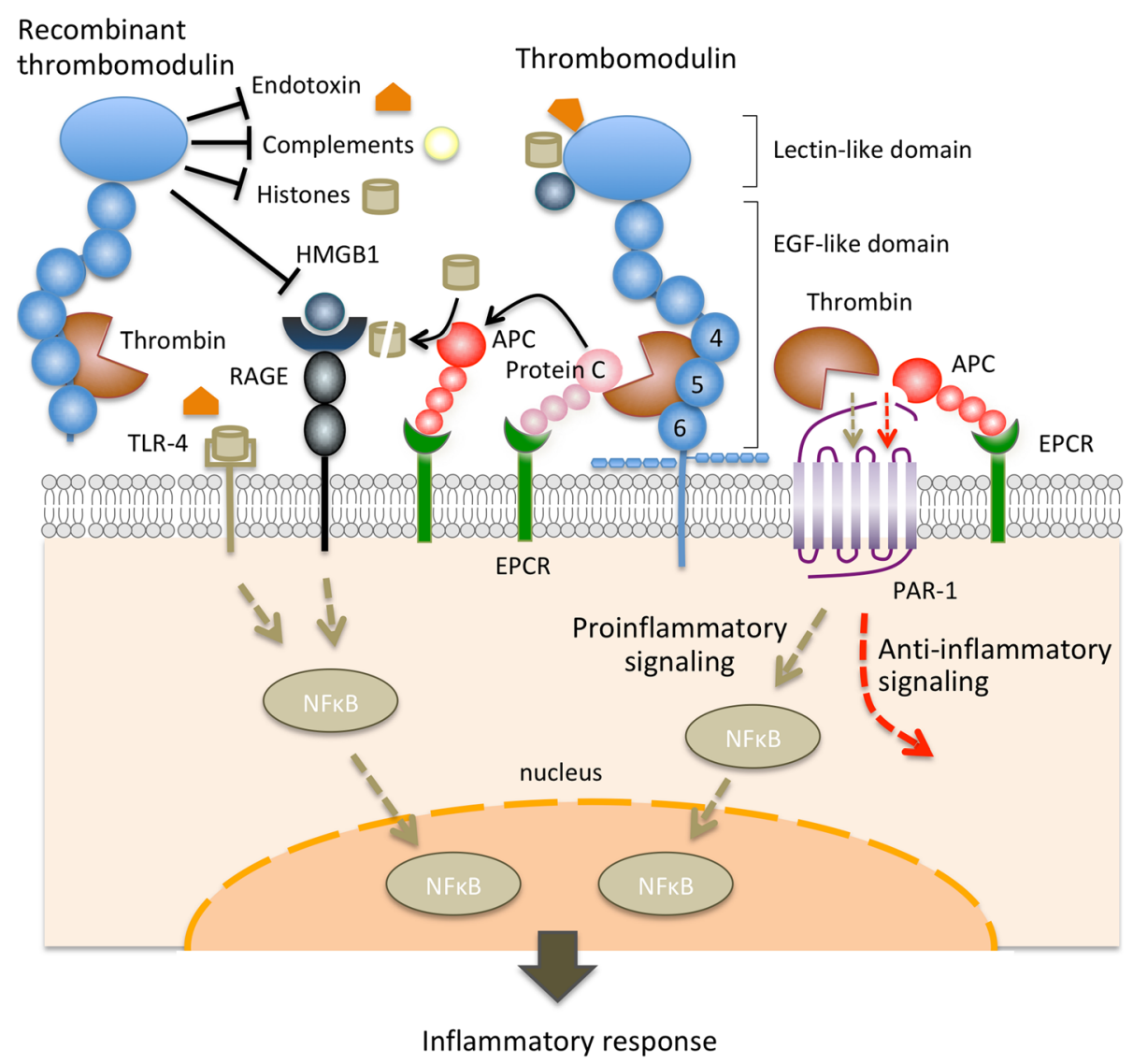

Fig. 1 Anti-inflammatory effects of thrombomodulin. The thrombin-thrombomodulin complex activates protein $C$ on the surface of endothelial cells, and this activation is facilitated by endothelial protein $C$ receptor (EPCR). Although thrombin initiates proinflammatory signaling by cleaving protease-activated receptor 1 (PAR1) (brown dotted arrow), activated protein C (APC) associated with EPCR cleaves PAR1 differently and initiates cell signaling that provides anti-inflammatory effects (red dotted arrow). The lectin-like domain of TM binds high mobility group box 1 (HMGB1) and inhibits its signaling via the receptor for advanced glycation end product (RAGE). The activation of RAGE by HMGB1 initiates the nuclear translocation of nuclear factor kappa B (NF-kB) that induces an inflammatory response. Similarly, the lectin-like domain of thrombomodulin blocks the interaction between Toll-like receptor 4 (TLR-4) and its ligands, such as endotoxin and histones, thereby inhibiting proinflammatory reactions

described in other reviews [10, 29, 30]. One of the pivotal trials in the management of septic coagulopathy was the recombinant APC (drotrecogin alfa [activated], Xigris ${ }^{\circ}$ ) study [31]. However, the most recent analysis of its efficacy and safety for use in septic patients led to its removal from clinical use [32]. The failure of the most recent recombinant APC study was due to multiple considerations including bleeding side effects, dosing, timing, and efficacy. Among all, the most important consideration is the patient selection. Anticoagulants such as recombinant APC should be effective only to the septic patients with coagulopathy or DIC [25]. Hence, subgroup analyses in large-scale RCTs have been performed to examine the effect of anticoagulants, including antithrombin and recombinant APC, in patients with sepsis and have reported trends toward a greater risk reduction in mortality among patients with DIC than among patients without DIC [33, 34].
Based on this background, a phase III, randomized, double-blind, parallel-group trial comparing rTM and heparin in 234 patients with DIC associated with infection or hematologic malignancy was performed in Japan [3]. A sub-group analysis for the septic DIC patients revealed that the absolute difference in mortality was $11.2 \%$ (rTM $21.4 \%$ vs. heparin $31.6 \%$ ), but the difference was not statistically significant $(95 \%$ confidence interval [CI] -9.1-29.4\%) [35]. However, since the primary endpoint, DIC resolution rate, was significantly better in the rTM group (difference 16.2\%, 95\% CI 3.3-29.1), the Japanese healthcare system approved rTM as a therapeutic agent for DIC in 2008. Subsequently, a multi-national RCT was conducted to examine the effect of rTM in sepsis with suspected DIC [36]. This phase IIb trial was performed in 233 ICUs in 17 countries, and a total of 750 patients with septic coagulopathy (thrombocytopenia with prolonged prothrombin time) were enrolled. The results 
revealed a $3.8 \%$ reduction in the absolute risk of death (rTM group: $17.8 \%$ vs. placebo group: $21.6 \%, P=0.273$ ). Though this study could not demonstrate a statistically significant survival benefit, it is noteworthy that coagulation biomarker levels such as D-dimer and thrombin-antithrombin complex (TAT) levels decreased in the rTM group compared to the placebo group. Hoppensteadt et al. [37] further analyzed data from this phase IIb trial and reported that prothrombin fragment 1.2 levels decreased by $16 \%$ from the baseline until day 7 in DIC patients treated with rTM, whereas the level increased by $8 \%$ in the placebo control. The phase IIb study also reported that the efficacy was more prominent in patients with prothrombin time ratio $>1.4$ and organ dysfunction.

Since its approval for the treatment of DIC in Japan, the clinical effectiveness of rTM has been extensively evaluated. Yamakawa et al. [38] collected data from 12 studies (838 patients from 3 RCTs and 571 patients from 9 observational studies) and reported that the relative risk of death was 0.81 (95\% CI 0.62-1.06) in the RCTs and 0.59 (95\% CI 0.45-0.77) in the observational studies. Although the meta-analysis reported a trend toward favorable outcomes, inconsistent results were also reported. For example, Tagami et al. [39] performed propensity score and instrumental variable analyses using a Japanese nationwide administrative database (matched cohort of 1140 pairs) and reported that the allcause mortality was $37.6 \%$ in the rTM treatment group vs. $37.0 \%$ in the control group (odds ratio [OR] 1.01, 95\% CI 0.93-1.10). This result demonstrated that treatment with rTM did not reduce mortality among patients with pneumonia-associated DIC. Since previous studies indicate that severe cases gain more benefit from anticoagulants, the survival benefit might have been observed if the study had targeted more critically ill patients [34, 40]. Subsequently, Yoshimura et al. [41] performed a post hoc analysis using data from a multicenter retrospective cohort study and reported that the administration of rTM was significantly associated with reduced mortality among patients with a high risk of death (APACHE II score 2429 , hazard ratio $[\mathrm{HR}] 0.281,95 \%$ CI $0.093-0.850, P=$ 0.025). In contrast, an association was not evident among lower-risk patients (APACHE II score < 24, HR 0.814, 95\% CI 0.351-1.884, $P=0.630$ ). They concluded that the survival benefit was obtained only in septic DIC patients with a high risk of death. Recently, Hayakawa et al. [42] collected data from 42 intensive care units and performed a propensity analysis of 452 matched pairs. The results revealed a significant association between rTM administration and a lower in-hospital mortality (OR 0.757, 95\% CI $0.574-0.999, P=0.049$ ). Regarding safety features, postmarketing surveillance of rTM among 2516 septic patients with DIC demonstrated that the frequency of critical bleeding was $2.6 \%$ [43], which did not differ from that in a phase III trial performed in Japan (rTM group $1.72 \%$, heparin group 2.61\%) [3]. Based on these results, the Japanese Society on Thrombosis and Hemostasis recommends the use of rTM for DIC [44], while Surviving Sepsis Campaign Guidelines [45] and Japanese Clinical Practice Guidelines [46] for the Management of Sepsis and Septic Shock postponed the recommendation in their 2016 version.

Following these studies, the efficacy and safety of rTM in the adult patients with sepsis and coagulation disorders have been examined in a multinational, randomized, placebo-controlled, double-blinded phase III trial named SCARLET (Sepsis Coagulopathy Asahi Recombinant LE Thrombomodulin). Accordingly, a $2.6 \%$ reduction in the absolute risk of 28-day mortality (rTM group $26.8 \%$ vs. placebo group $29.4 \%$ ) was reported in a total of 800 sepsis patients (rTM group, $n=395$; placebo group, $n=405$ ) with coagulopathy (platelet count < $150 \times 10^{9} / \mathrm{L}$ and prothrombin time ratio > 1.4) and cardiovascular and/or respiratory failure, but the difference did not reach statistical difference [4]. However, it was also reported that approximately $20 \%$ of patients did not fulfill the entry criterion at baseline after initial confirmation of eligibility and that $5.4 \%$ (95\% CI, $-1.68-12.48)$ reduction in the absolute risk of 28-day mortality was observed in patients fulfilled the entry criterion at baseline. Following the SCARLET study, Yamakawa et al. [5] updated the meta-analysis for rTM and reported that in 1762 patients enrolled, approximately 13\% reduction in the risk of mortality was observed in the rTM group, but the difference was not significant (relative risk $0.87,95 \%$ CI $\left.0.74-1.03, P=0.10, I^{2}=0 \%\right)$. It is noteworthy that all clinical trials except one showed a similar trend in favor of rTM in terms of survival and a significant improvement in coagulation parameters. The finding that the effects of rTM were more prominent in patients with coagulation disorders will also support the potential of this anticoagulant. Although TM is one of the most important physiologic anticoagulants, and the effect of externally administered rTM has long been examined through the clinical trials and practice [47], further clinical study is required to prove its efficacy. We have to keep in mind that sepsis is a complex mix of heterogeneous components and therefore, it may not be possible to find a one-fit-all therapeutic agent. Like other clinical trials for the anticoagulants, the patient selection should be the key to the success in the rTM study.

\section{The efficacy of rTM in hematological malignancies}

In contrast to sepsis-associated DIC where organ dysfunction due to microthrombosis occurs, DIC associated with hematological malignancies exhibits bleeding symptoms. Consumptive coagulopathy and profound fibrinolysis are thought to be responsible for bleeding 
symptoms in this previously described "enhanced-fibrinolytic-type" DIC [48]. Thus, targeted inhibition of both coagulation and fibrinolysis is important in managing enhanced-fibrinolytic-type DIC, such as acute promyelocytic leukemia (APL)-associated DIC. Specifically, nafamostat mesylate, a serine protease inhibitor that has both antithrombotic and antifibrinolytic effects, or combined therapy of a heparinoid drug and tranexamic acid, is effective against bleeding symptoms in enhanced-fibrinolytic-type DIC $[49,50]$. However, in patients with complex coagulopathic states, antifibrinolytic agents have the potential risk of thrombotic complications due to fibrinolytic shutdown [51].

A clinical trial of rTM for the treatment of DIC with underlying hematologic malignancy or infection was conducted in Japan with unfractionated heparin as a control [3]. The DIC resolution rate was $66.1 \%$ in the rTM treatment group and $49.9 \%$ in the heparin treatment group. Moreover, bleeding symptoms became significantly better in the rTM group. Bleeding-related adverse events during the 7 days of treatment were $43.1 \%$ in the rTM group and $56.5 \%$ in the heparin group. Interestingly, the superiority of rTM was shown to be greater in the hematological malignancy patients than in the infection patients in this clinical trial. Factors responsible for the lower likelihood of adverse bleeding events have been proposed. One is rTM only exhibits an anticoagulant effect in the presence of thrombin [52], and antifibrinolytic TAFI, which can be activated by the thrombin-TM complex, may contribute to the suppression of fibrinolysis-related bleeding symptoms [53].

The safety and efficacy of rTM in patients with DIC and hematological malignancy $(n=1032)$ have also been reported from post-marketing surveillance [54]. In DIC patients who had bleeding symptoms at baseline, 55\% were assessed as bleeding disappeared or improved after rTM treatment. DIC-related coagulation and fibrinolytic markers were also improved even in patients whose clinical course of underlying disease was unchanged or exacerbated. This is interesting because DIC generally depends on the underlying disease in cases of hematologic malignancy.

In a retrospective analysis of acute myeloblastic leukemia ( $n=103$, of whom 47 had DIC), rTM was reported to improve both DIC and the survival outcomes $(P=0.016)$ [55]. The lectin-like domain of rTM may confer this survival benefit through inhibiting HMGB1. HMGB1 is abundantly expressed in several leukemia cells, and inhibition of HMGB1 enhances the sensitivity to chemotherapy of leukemia cells [56]. The epidermal growth factor-like domain of rTM may also confer protection against leukemia by inducing growth arrest and differentiation of leukemia cells [57]. Thus, rTM might be promising both for the control of DIC and for the direct anti-leukemic effect.
All-trans retinoic acid (ATRA) has transformed the care of patients with APL. In the present context, ATRA inhibits intravascular coagulation not only by decreasing the expression of tissue factor in APL cells but also by increasing the expression of TM. ATRA also inhibits fibrinolysis by decreasing annexin II expression [58]. However, in some cases, DIC can be exacerbated by concurrent differentiation syndrome after ATRA therapy or tumor-lysis after chemotherapy. In such cases, rTM is effective and may reduce bleeding-related death in APL patients [59], possibly by enhancing the anti-fibrinolytic and anti-leukemic effects of ATRA in APL cells [60]. Thus, the administration of rTM for AML is promising in terms of both its anti-DIC and anti-APL cell effects.

In the setting of hematopoietic stem cell transplantation, rTM is helpful for the treatment of transplant-related serious complications, such as thrombotic microangiopathy [61], veno-occlusive disease (also known as sinusoidal obstruction syndrome) [62], and graft-versus-host disease (GVHD) [63]. Although the protective mechanisms of rTM in these complications remain to be fully elucidated, anti-inflammatory, as well as anticoagulant effects of rTM, may be involved. Furthermore, APC-mediated PAR activation may help in expanding regulatory $\mathrm{T}$ cells and in mitigating GVHD [64]. Protective roles of TM in GVHD are further supported by a cohort study showing that single-nucleotide polymorphisms within the TM gene predict mortality in patients with GVHD [65].

\section{The effects of rTM in solid tumor-associated DIC}

TM is known to be expressed by tumor cells [66]. TM exhibits anti-cancer properties by acting on various steps in the oncologic pathway including affecting cancer cell proliferation, blocking tumor invasion, and metastasis. The underlying mechanisms are different depending on the type of malignancy, but one of the mechanisms suggested is a suppression of epithelial-mesenchymal transition, which can make cancer cells more susceptible to chemotherapy [67]. Another suggested mechanism is through the thrombin-PAR1 pathway [68]. Interestingly, low TM expression in tumor cells is correlated with poor prognosis, and up-regulation of TM expression by atorvastatin, an HMG-CoA reductase inhibitor, diminishes the tumorigenic capability of lung cancer cells [69]. In spite of these experimental findings, few human cancer trials of rTM have been performed, and most of them have been in the context of cancer-induced DIC.

In cases of DIC associated with solid tumors, the cancer is usually in the terminal stages and is often associated with systemic metastasis. DIC treatment should be considered in such cases when control of DIC can potentially extend survival. Reports on the use of rTM for solid tumor-associated DIC are still sparse [70]. Tamura 
et al. [71] reported a prospective one-arm study of 101 DIC patients (lung, stomach, breast, and other cancers). The DIC resolution rate with rTM therapy was $34.0 \%$, while DIC scores improved in $55.2 \%$ of patients and worsened in $22.9 \%$. The incidence of hemorrhage, mainly gross hematuria, related to rTM was $12.9 \%$ until day 28. Severe hemorrhage related to rTM did not occur. In 26 patients who could not be treated with anticancer agents, the DIC resolution rate and the rate of improvement in DIC scores were $27 \%$ and $40 \%$, respectively. Among them, anti-cancer therapy could be restarted in four patients in whom DIC was controlled with rTM. These results suggest the potential benefit of rTM treatment for the solid tumor-associated DIC. In prostate cancer, malignant melanoma, vascular tumors, and some other cancers, life-threatening bleeding symptoms may occur due to the enhanced-fibrinolytic-type DIC [72]. In such cases, rTM, nafamostat mesylate, or combined therapy of heparinoid drug and tranexamic acid is thought to be useful in managing DIC-related bleeding symptoms.

\section{The efficacy of rTM in respiratory diseases}

ARDS is a deadly complication of various diseases which causes severe lung inflammation. In addition to supportive care, there have not been any therapeutic advances despite the use of extracorporeal membrane oxygenation. The identification of the crucial role of HMGB1 in this regard once again brings rTM as an interesting therapeutic prospect in the ARDS setting [73]. HMGB1 levels in the lung were elevated in ARDS mice, and rTM administration decreased the development of ARDS that correlated with an increased T-reg cell population [74]. Other researchers evaluated the possible therapeutic effect of rTM in attenuating animal models of ARDS [75, 76] and reported HMGB1 and proinflammatory cytokine levels were significantly lowered by rTM administration. These findings confirm the anti-inflammatory effect of rTM by potential inhibition of HMGB1 pathway, which may subsequently be translated to better survival outcome.

The bidirectional interaction between inflammation and coagulation can play an important role in the pathophysiology of progressive pulmonary fibrosis. Both early- and late-phase administration of rTM suppressed the fibrotic process possibly modulating the thrombin effects and also by blocking the action of HMGB1 in a mouse model of bleomycin-induced lung fibrosis [77]. Recently, the effects of rTM on acute exacerbation of idiopathic pulmonary fibrosis (IPF) have attracted more attention. In addition to the injury of type II alveolar epithelial cells and capillary endothelial cells, the activation of coagulation is revealed to deeply be involved in the pathogenesis of this critical condition [78]. Furthermore, increased levels of soluble TM, plasminogen activator inhibitor1, fibrin/fibrinogen degradation products, D-dimer, and thrombin-antithrombin complex were reported in patients with acute exacerbation of IPF [79, 80]. Based on these observations, the effects of rTM have been examined in clinical cases. First, Kataoka et al. [81] examined the efficacy of rTM in patients with acute exacerbation of IPF in a historical cohort study. Twenty patients treated with rTM for about 6 days showed significantly improved three-month mortality compared to the control group (OR 0.219; 95\% CI $0.049-0.978 ; P=0.047)$. A larger study with $45 \mathrm{pa}$ tients treated with rTM confirmed similar findings (OR 0.250; 95\%CI 0.091-0.685) [82]. More recently, the result of a single-arm open-label multicenter cohort study was reported, in which 90-day survival rate of in rTM-treated group was 66.7\% (26/39) and that in the historical control was $47.5 \%(29 / 61)$ (adjusted HR $0.453 ; 95 \%$ CI $0.237-0.864 ; \quad P=0.0163$ ) [83]. Though the concept of treating acute exacerbation of IPF with rTM is interesting, the studies are still exploratory. High-quality evidence is needed to further support additional therapeutic applications of rTM.

\section{The roles of TM in renal diseases}

The most common disease in relation to TM is hemolytic uremic syndrome (HUS) [84], which consists of the triad of micro-angiopathic hemolytic anemia, thrombocytopenia, and renal failure, and should be distinguished from DIC [85]. Atypical HUS, which is not triggered by Shiga-toxin, constitutes about $10 \%$ of all cases and has a poor prognosis. About half of atypical HUS cases are caused by the genetic mutations in complement regulation. Delvaeye et al. [13] reported that TM can accelerate factor I-mediated inactivation of $\mathrm{C} 3 \mathrm{~b}$ and, by activating procarboxypeptidase $\mathrm{B}$, speeds up the inactivation of anaphylatoxins $\mathrm{C} 3 \mathrm{a}$ and $\mathrm{C} 5 \mathrm{a}$ in vitro. Consistent with these findings, $5 \%$ of atypical HUS cases are caused by mutations in the TM gene [13]. Based on these findings, rTM was evaluated in three Japanese children with HUS and mitigated thrombocytopenia, hemolysis, and renal dysfunction in the three patients and decreased complement activation in one of the patients [86]. All the patients made good recovery without any neurological sequelae, abnormal renal dysfunction, or adverse effects from rTM. In an experiment with severe and moderate HUS-model mice injected with Shiga toxin and lipopolysaccharide, it was noted that rTM administration decreased mortality [87]. Hemoglobin, platelet counts, inflammatory biomarkers, biomarkers of endothelial injury, and mesangiolysis scores were improved within $24 \mathrm{~h}$ after the administration of rTM. Animal studies have also demonstrated protective roles of 
TM in the setting of pyelonephritis [88], and protective effects of rTM in a rat model of anti-glomerular basement membrane glomerulonephritis [89].

\section{The roles of TM in cardiovascular diseases}

TM is widely distributed throughout the vasculature. However, in some organs, its expression is restricted in a vascular-bed-specific manner. The brain is one such example, and TM is sparse or even absent in blood vessels of the putamen in young subjects [90]. In mice, endothelium-specific loss of TM results in increased fibrin deposition in the lungs and heart, but not in the brain, suggesting that molecules other than TM contribute to brain vascular patency under normal conditions. Nevertheless, exogenously administered TM reduces the infarct volume in murine

Table 1 Summaries of RCTs and observational studies on rTM

\begin{tabular}{|c|c|c|c|c|}
\hline \multicolumn{5}{|l|}{ Summary of RCTs } \\
\hline \multirow[t]{2}{*}{ Disease } & \multirow[t]{2}{*}{ Source } & \multicolumn{2}{|l|}{ Intervention } & \multirow[t]{2}{*}{ Mortality (\%) } \\
\hline & & rTM & Control & \\
\hline \multirow[t]{4}{*}{ Sepsis } & Aikawa et al. [35] & 0.06 mg/kg/day for 6 days $(n=42)$ & UFH $(n=38)$ & $21.4 \%$ vs $31.6 \% \quad 73.2 \%$ vs \\
\hline & Vincent et al. [36] & $0.06 \mathrm{mg} / \mathrm{kg} /$ day for 6 days $(n=370)$ & Placebo $(n=371)$ & $17.8 \%$ vs $21.6 \% \quad 28.9 \%$ vs \\
\hline & Hagiwara et al. [97] & $0.06 \mathrm{mg} / \mathrm{kg} /$ day up to 6 days $(n=45)$ & w/o rTM $(n=47)$ & $17.0 \%$ vs $15.6 \% * 90.7 \%$ v \\
\hline & Vincent et al. [4] & 0.06 mg/kg/day for 6 days $(n=395)$ & Placebo $(n=405)$ & $26.8 \%$ vs $29.4 \% \quad N / A$ \\
\hline Hemotologic malignancy & Saito et al., [3] & 0.06 mg/kg/day for 6 days $(n=64)$ & UFH $(n=61)$ & $17.2 \%$ vs $18.0 \% * 65.6 \%$ v \\
\hline \multicolumn{5}{|c|}{ Summary of observational studies } \\
\hline \multirow[t]{2}{*}{ Disease } & \multirow[t]{2}{*}{ Source } & \multicolumn{2}{|l|}{ Intervention } & \multirow[t]{2}{*}{ Results (95\% Cl) } \\
\hline & & rTM & Control & \\
\hline \multirow[t]{10}{*}{ Sepsis } & Ohryoji et al. [38] & 0.06 mg/kg/day for 6 days $(n=17)$ & $\begin{array}{l}\text { w/o rTM }(n=16) \\
\text { historical control }\end{array}$ & $\begin{array}{l}\text { Mortality rate } \\
23.5 \% \text { vs } 50.0 \%\end{array}$ \\
\hline & Yada et al. [38] & 0.06 mg/kg/day for 6 days $(n=12)$ & w/o rTM $(n=16)$ & $\begin{array}{l}30-\text { day mortality rate } \\
25.0 \% \text { vs } 18.8 \%\end{array}$ \\
\hline & Kudo et al. [38] & 0.06 mg/kg/day for 6 days $(n=30)$ & $\begin{array}{l}\text { w/o rTM }(n=23) \\
\text { historical control }\end{array}$ & $\begin{array}{l}\text { 30-day mortality rate } \\
* 10.0 \% \text { vs } 34.8 \%\end{array}$ \\
\hline & Umegaki et al. [38] & 0.06 mg/kg/day for 7 days $(n=33)$ & $\begin{array}{l}\text { Danaparoid }(n=40) \\
\text { historical control }\end{array}$ & $\begin{array}{l}\text { 28-day mortality } \\
\text { HR } 0.72(0.31-1.66)\end{array}$ \\
\hline & Yamakawa et al. [98] & $0.06 \mathrm{mg} / \mathrm{kg} /$ day for 6 days $(n=68)$ & w/o rTM $(n=94)$ & $\begin{array}{l}\text { In-hospital mortality } \\
{ }^{*} H R 0.45(0.26-0.77)\end{array}$ \\
\hline & Kato et al. [99] & 0.06 mg/kg/day for 7 days $(n=12)$ & w/o rTM $(n=23)$ & $\begin{array}{l}\text { 28-day mortality } \\
\text { *HR } 0.384 \text { (0.088-0.904) }\end{array}$ \\
\hline & Sawano et al. [38] & $0.06 \mathrm{mg} / \mathrm{kg} / \mathrm{day}$ for 6 days $(n=51)$ & w/o rTM $(n=60)$ & $\begin{array}{l}\text { 28-day mortality } \\
\text { *HR } 0.28(0.11-0.72)\end{array}$ \\
\hline & Yamato et al. [100] & 0.06 mg/kg/day for 6 days $(n=14)$ & $\begin{array}{l}\text { w/o rTM }(n=8) \\
\text { historical control }\end{array}$ & $\begin{array}{l}\text { 28-day mortality rate } \\
* 14.3 \% \text { vs } 62.5 \%\end{array}$ \\
\hline & Tagami et al. [39] & 0.06 mg/kg/day for 6 days $(n=1140)$ & w/o rTM $(n=1140)$ & $\begin{array}{l}\text { 28-day mortality } \\
\text { OR } 1.01(0.93-1.10)\end{array}$ \\
\hline & Hayakawa et al. [42] & 0.06 mg/kg/day for 6 days $(n=452)$ & w/o rTM $(n=452)$ & $\begin{array}{l}\text { In-hospital mortality } \\
{ }^{*} \text { OR } 0.757 \text { (0.574-0.999) }\end{array}$ \\
\hline $\mathrm{AML}$ & Takezako et al. [55] & 0.06 mg/kg/day for 6 days $(n=14)$ & $\mathrm{LMWH}(n=33)$ & $\begin{array}{l}\text { Estimated overall survival } \\
\text { *Superior in rTM }\end{array}$ \\
\hline \multirow[t]{2}{*}{ AE of IPF } & Kataoka et al. [81] & 0.06 mg/kg/day for 6 days $(n=20)$ & $\begin{array}{l}\text { w/o rTM }(n=20) \\
\text { historical control }\end{array}$ & $\begin{array}{l}\text { 3-month mortality } \\
{ }^{*} \text { OR } 0.219(0.049-0.978)\end{array}$ \\
\hline & Sakamoto et al. [82] & 0.06 mg/kg/day for 6 days $(n=45)$ & $\begin{array}{l}\text { w/o rTM }(n=35) \\
\text { historical control }\end{array}$ & $\begin{array}{l}\text { 3-month mortality } \\
{ }^{*} \text { OR } 0.250(0.091-0.685)\end{array}$ \\
\hline AE of $\| P$ & Arai et al. [83] & $0.06 \mathrm{mg} / \mathrm{kg} /$ day for 6 days $(n=39)$ & $\begin{array}{l}\text { w/o rTM }(n=61) \\
\text { historical control }\end{array}$ & $\begin{array}{l}\text { 90-day mortality } \\
\text { *HR } 0.453(0.237-0.864)\end{array}$ \\
\hline
\end{tabular}

DIC disseminated intravascular coagulation, $r T M$ recombinant thrombomodulin, UFH unfractionated heparin, N/A not available, $C I$ confidence interval, $A M L$ acute myeloblastic leukemia, $A E$ acute exacerbation, IPF idiopathic pulmonary fibrosis, IIP idiopathic interstitial pneumonia, $L M W H$ low molecular weight heparin, $H R$ hazard ratio, OR odds ratio *Statistically significant 
stroke models, possibly through its anti-inflammatory and anticoagulant actions [91, 92]. These findings suggest the potency of rTM for the treatment of acute ischemic stroke.

TM is expressed on the luminal surface of coronary arteries under normal physiologic conditions. However, TM expression is locally downregulated in atherosclerotic lesions of coronary arteries [93]. In atherogenic mice, exogenously administered TM reduces atherosclerosis and neointima formation in a thrombin-dependent manner [94]. Thus, rTM may be useful to limit the progression of atherosclerosis, although some difficulties are inherent in long-term parenteral administration of rTM.

There is controversy regarding the significance of endogenous soluble TM levels as a biomarker of cardiovascular diseases. In healthy individuals, a high concentration of soluble TM may be associated with a low risk of coronary heart disease [95]. In contrast, a high concentration of soluble TM may predict fatal outcomes of patients who have already had a brain infarction [96]. Interpretation of soluble TM values can be difficult because a high concentration of soluble TM indicates increased expression of $\mathrm{TM}$ on the surface of healthy endothelial cells or increased release of soluble fragments of TM from damaged endothelial cells. The former may be associated with good outcomes because of the cytoprotective effects of this protein, whereas the latter may be associated with poor outcomes because of endothelial damage. Therefore, soluble TM values should be interpreted according to the vascular history of patients [96].

\section{Conclusion}

The discovery of TM has facilitated a new era of research focusing on the crosstalk between coagulation and inflammation. It has also formed the basis of a therapeutic model that could be targeted to clinical states associated with endothelial dysfunction. The earliest trials have been in the classical disease state of DIC, where the link between inflammation and coagulation has been extensively studied (Table 1). The success of rTM in the DIC setting, notably with the tolerable side effects profile, divides an important therapeutic approach in this complex coagulopathy. Additional studies continue to determine the optimal role for rTM in the therapeutic armamentarium for disease conditions where endothelial dysfunction coupled with proinflammatory states exist. However, further randomized controlled trials with adequate patient numbers are required to better define the therapeutic role of rTM. On a related note, standardization of soluble TM measurements is required prior to its use as a marker of endothelial injury, because the interpretation of the soluble TM level remains controversial.

\section{Abbreviations}

APACHE II score: Acute physiology and chronic health evaluation II score; APC: Activated protein C; APL: Acute promyelocytic leukemia; ARDS: Acute respiratory distress syndrome; ATRA: All-trans retinoic acid; Cl: Confidence interval; DIC: Disseminated intravascular coagulation; EPCR: Endothelial protein C receptor; GVHD: Graft-versus-host disease; HMGB1: High mobility group box 1; HR: Hazard ratio; HUS: Hemolytic uremic syndrome; OR: Odds ratio; PAR1: Protease-activated receptor 1; RCT: Randomized controlled trial; rTM: Recombinant thrombomodulin; TAFI: Thrombin activatable fibrinolysis inhibitor; TAT: Thrombin-antithrombin complex; TM: Thrombomodulin

\section{Acknowledgements \\ Not applicable. \\ Authors' contributions \\ All authors conducted the literature search and edited the manuscript. All authors read and approved the final manuscript.}

Funding

No funding applies.

Availability of data and materials

Not applicable.

Ethics approval and consent to participate Not applicable.

\section{Consent for publication}

Not applicable.

\section{Competing interests}

TI, HA and TI received research grants from Asahi Kasei Pharma, a pharmaceutical company manufacturing recombinant thrombomodulin. JHL is a member of research steering committees or advisory boards for Boehringer-Ingelheim, CSL Behring, Instrumentation Laboratories,

Octapharma, and Merck. The funds are for academic promotion and are not directly related to this study. The remaining authors declare that they have no competing interests.

\section{Author details}

${ }^{1}$ Department of Systems Biology in Thromboregulation, Kagoshima University Graduate School of Medical and Dental Sciences, 8-35-1 Sakuragaoka, Kagoshima 890-8544, Japan. ²Department of Haematology, Manchester University Hospitals NHS Foundation Trust, Manchester, UK. ${ }^{3}$ Third Department of Internal Medicine, Kanazawa University Graduate School of Medical Science, Kanazawa, Japan. ${ }^{4}$ Department of Anesthesiology, Critical Care and Surgery, Duke University School of Medicine, Durham, NC, USA. ${ }^{5}$ Department of Emergency and Disaster Medicine, Juntendo University Graduate School of Medicine, Tokyo, Japan.

Received: 19 May 2019 Accepted: 23 July 2019

Published online: 15 August 2019

\section{References}

1. Esmon NL, Owen WG, Esmon CT. Isolation of a membrane-bound cofactor for thrombin-catalyzed activation of protein C. J Biol Chem. 1982;257(2): 859-64.

2. Loghmani $\mathrm{H}$, Conway EM. Exploring traditional and nontraditional roles for thrombomodulin. Blood. 2018;132(2):148-58.

3. Saito H, Maruyama I, Shimazaki S, et al. Efficacy and safety of recombinant human soluble thrombomodulin (ART-123) in disseminated intravascular coagulation: results of a phase III, randomized, double-blind clinical trial. J Thromb Haemost. 2007:5(1):31-41.

4. Vincent JL, Francois B, Zabolotskikh I, et al. Effect of a recombinant human soluble thrombomodulin on mortality in patients with sepsis-associated coagulopathy: the SCARLET randomized clinical trial. JAMA. 2019;321(20): 1993-2002.

5. Yamakawa K, Murao S, Aihara M. Recombinant human soluble thrombomodulin in sepsis-induced coagulopathy: an updated systematic review and meta-analysis. Thromb Haemost. 2019;119(1):56-65. 
6. Fuentes-Prior $P$, Iwanaga $Y$, Huber $R$, et al. Structural basis for the anticoagulant activity of the thrombin-thrombomodulin complex. Nature. 2000;404(6777):518-25

7. Isermann B, Hendrickson SB, Zogg M, et al. Endothelium-specific loss of murine thrombomodulin disrupts the protein $\mathrm{C}$ anticoagulant pathway and causes juvenile-onset thrombosis. J Clin Investig. 2001;108(4):537-46.

8. Mohan Rao LV, Esmon CT, Pendurthi UR. Endothelial cell protein C receptor: a multiliganded and multifunctional receptor. Blood. 2014;124(10):1553-62.

9. Riewald M, Petrovan RJ, Donner A, et al. Activation of endothelial cell protease activated receptor 1 by the protein C pathway. Science. 2002; 296(5574):1880-2.

10. Conway EM. Thrombomodulin and its role in inflammation. Semin Immunopathol. 2012;34(1):107-25.

11. Conway EM, Van de Wouwer M, Pollefeyt $\mathrm{S}$, et al. The lectin-like domain of thrombomodulin confers protection from neutrophil-mediated tissue damage by suppressing adhesion molecule expression via nuclear factor kappaB and mitogen-activated protein kinase pathways. J Exp Med. 2002; 196(5):565-77.

12. Van de Wouwer M, Plaisance S, De Vriese A, et al. The lectin-like domain of thrombomodulin interferes with complement activation and protects against arthritis. J Thromb Haemost. 2006;4(8):1813-24.

13. Delvaeye $M$, Noris $M$, De Vriese $A$, et al. Thrombomodulin mutations in atypical hemolytic-uremic syndrome. N Engl J Med. 2009;361(4): 345-57.

14. Ito T, Kakihana Y, Maruyama I. Thrombomodulin as an intravascular safeguard against inflammatory and thrombotic diseases. Expert Opin Ther Targets. 2016;20(2):151-8.

15. Shi CS, Shi GY, Hsiao HM, et al. Lectin-like domain of thrombomodulin binds to its specific ligand Lewis $Y$ antigen and neutralizes lipopolysaccharide-induced inflammatory response. Blood. 2008;112(9): 3661-70.

16. Boehme MW, Deng $Y$, Raeth $U$, et al. Release of thrombomodulin from endothelial cells by concerted action of TNF-alpha and neutrophils: in vivo and in vitro studies. Immunology. 1996;87(1):134-40.

17. Takano S, Kimura S, Ohdama S, et al. Plasma thrombomodulin in health and diseases. Blood. 1990;76(10):2024-9.

18. Gando S, Nakanishi Y, Kameue T, et al. Soluble thrombomodulin increases in patients with disseminated intravascular coagulation and in those with multiple organ dysfunction syndrome after trauma: role of neutrophil elastase. J Trauma. 1995:39(4):660-4.

19. Ohlin AK, Larsson K, Hansson M. Soluble thrombomodulin activity and soluble thrombomodulin antigen in plasma. J Thromb Haemost. 2005;3(5): 976-82.

20. Uehara S, Gotoh K, Handa H. Separation and characterization of the molecular species of thrombomodulin in the plasma of diabetic patients. Thromb Res. 2001;104(5):325-32.

21. Suzuki K, Kusumoto $H$, Deyashiki $Y$, et al. Structure and expression of human thrombomodulin, a thrombin receptor on endothelium acting as a cofactor for protein C activation. EMBO J. 1987;6(7):1891-7.

22. Mohri M, Sugimoto $E$, Sata $M$, et al. The inhibitory effect of recombinant human soluble thrombomodulin on initiation and extension of coagulation--a comparison with other anticoagulants. Thromb Haemost. 1999:82(6):1687-93.

23. Dhainaut JF, Yan SB, Margolis BD, et al. Drotrecogin alfa (activated) (recombinant human activated protein $\mathrm{C}$ ) reduces host coagulopathy response in patients with severe sepsis. Thromb Haemost. 2003;90(4): 642-53.

24. Arishima $T$, Ito $T$, Yasuda $T$, et al. Circulating activated protein $C$ levels are not increased in septic patients treated with recombinant human soluble thrombomodulin. Thromb J. 2018;16:24

25. Umemura $Y$, Yamakawa $K$, Ogura $H$, et al. Efficacy and safety of anticoagulant therapy in three specific populations with sepsis: a metaanalysis of randomized controlled trials. J Thromb Haemost. 2016;14(3): $518-30$

26. Moll S, Lindley C, Pescatore $S$, et al. Phase I study of a novel recombinant human soluble thrombomodulin, ART-123. J Thromb Haemost. 2004;2(10): 1745-51.

27. Tsuruta K, Yamada Y, Serada M, et al. Model-based analysis of covariate effects on population pharmacokinetics of thrombomodulin alfa in patients with disseminated intravascular coagulation and normal subjects. J Clin Pharmacol. 2011;51(9):1276-85
28. Hayakawa M, Kushimoto S, Watanabe E, et al. Pharmacokinetics of recombinant human soluble thrombomodulin in disseminated intravascular coagulation patients with acute renal dysfunction. Thromb Haemost. 2017; 117(5):851-9.

29. Iba T, Levy JH. Inflammation and thrombosis: roles of neutrophils, platelets and endothelial cells and their interactions in thrombus formation during sepsis. J Thromb Haemost. 2018;16(2):231-41.

30. Levi M, Van Der Poll T. Thrombomodulin in sepsis. Minerva Anestesiol. 2013; 79(3):294-8

31. Bernard GR, Vincent J-L, Laterre P-F, et al. Efficacy and safety of recombinant human activated protein C for severe Sepsis. N Engl J Med. 2001;344(10): 699-709.

32. Ranieri VM, Thompson BT, Barie PS, et al. Drotrecogin alfa (activated) in adults with septic shock. N Engl J Med. 2012;366(22):2055-64.

33. Kienast J, Juers $M$, Wiedermann $C J$, et al. Treatment effects of highdose antithrombin without concomitant heparin in patients with severe sepsis with or without disseminated intravascular coagulation. J Thromb Haemost. 2006;4(1):90-7

34. Dhainaut JF, Yan SB, Joyce DE, et al. Treatment effects of drotrecogin alfa (activated) in patients with severe sepsis with or without overt disseminated intravascular coagulation. J Thromb Haemost. 2004;2(11): 1924-33.

35. Aikawa N, Shimazaki S, Yamamoto $Y$, et al. Thrombomodulin alfa in the treatment of infectious patients complicated by disseminated intravascular coagulation: subanalysis from the phase 3 trial. Shock. 2011;35(4):349-54

36. Vincent $\mathrm{J}$, Ramesh MK, Ernest D, et al. A randomized, double-blind, placebo-controlled, phase $2 \mathrm{~b}$ study to evaluate the safety and efficacy of recombinant human soluble thrombomodulin, ART-123, in patients with sepsis and suspected disseminated intravascular coagulation. Crit Care Med. 2013;41(9):2069-79.

37. Hoppensteadt D, Tsuruta K, Cunanan J, et al. Thrombin generation mediators and markers in sepsis-associated coagulopathy and their modulation by recombinant thrombomodulin. Clin Appl Thromb Hemost. 2014;20(2):129-35.

38. Yamakawa $\mathrm{K}$, Aihara M, Ogura $\mathrm{H}$, et al. Recombinant human soluble thrombomodulin in severe sepsis: a systematic review and meta-analysis. J Thromb Haemost. 2015;13(4):508-19.

39. Tagami $T$, Matsui $H$, Horiguchi $H$, et al. Recombinant human soluble thrombomodulin and mortality in severe pneumonia patients with sepsisassociated disseminated intravascular coagulation: an observational nationwide study. J Thromb Haemost. 2015;13(1):31-40.

40. Freeman BD, Zehnbauer BA, Buchman TG. A meta-analysis of controlled trials of anticoagulant therapies in patients with sepsis. Shock. 2003; 20(1):5-9.

41. Yoshimura J, Yamakawa K, Ogura H, et al. Benefit profile of recombinant human soluble thrombomodulin in sepsis-induced disseminated intravascular coagulation: a multicenter propensity score analysis. Crit Care. 2015;19:78.

42. Hayakawa M, Yamakawa K, Saito S, et al. Recombinant human soluble thrombomodulin and mortality in sepsis-induced disseminated intravascular coagulation. A multicentre retrospective study. Thromb Haemost. 2016; 115(6):1157-66.

43. Mimuro J, Takahashi H, Kitajima I, et al. Impact of recombinant soluble thrombomodulin (thrombomodulin alfa) on disseminated intravascular coagulation. Thromb Res. 2013;131(5):436-43.

44. Wada H, Japanese Society of Thrombosis Hemostasis DICs, Okamoto K, et al. Addition of recommendations for the use of recombinant human thrombomodulin to the "Expert consensus for the treatment of disseminated intravascular coagulation in Japan". Thromb Res 2014; 134(4): 924-925.

45. Rhodes A, Evans LE, Alhazzani W, et al. Surviving Sepsis Campaign: International Guidelines for Management of Sepsis and Septic Shock: 2016. Intensive Care Med. 2017:43(3):304-77.

46. Nishida O, Ogura H, Egi M, et al. The Japanese Clinical Practice Guidelines for Management of Sepsis and Septic Shock 2016 (J-SSCG 2016). Acute Med Surg. 2018;5(1):3-89.

47. Iba T, Thachil J. Present and future of anticoagulant therapy using antithrombin and thrombomodulin for sepsis-associated disseminated intravascular coagulation: a perspective from Japan. Int J Hematol. 2016: 103(3):253-61. 
48. Asakura $\mathrm{H}$. Classifying types of disseminated intravascular coagulation: clinical and animal models. J Intensive Care. 2014;2(1):20.

49. Takahashi T, Suzukawa M, Akiyama M, et al. Systemic AL amyloidosis with disseminated intravascular coagulation associated with hyperfibrinolysis. Int J Hematol. 2008:87(4):371-4.

50. Ontachi $Y$, Asakura $H$, Arahata $M$, et al. Effect of combined therapy of danaparoid sodium and tranexamic acid on chronic disseminated intravascular coagulation associated with abdominal aortic aneurysm. Circ J. 2005;69(9):1150-3.

51. Charytan C, Purtilo D. Glomerular capillary thrombosis and acute renal failure after epsilon-amino caproic acid therapy. N Engl J Med. 1969;280(20): 1102-4.

52. Mohri M, Gonda Y, Oka M, et al. The antithrombotic effects of recombinant human soluble thrombomodulin (rhsTM) on tissue factor-induced disseminated intravascular coagulation in crab-eating monkeys (Macaca fascicularis). Blood Coagul Fibrinolysis. 1997;8(5):274-83.

53. Toh $\mathrm{CH}$. Characterization of thrombin activatable fibrinolysis inhibitor in normal and acquired haemostatic dysfunction. Blood Coagul Fibrinolysis. 2003;14(Suppl 1):S69-71.

54. Asakura $H$, Takahashi $H$, Tsuji $H$, et al. Post-marketing surveillance of thrombomodulin alfa, a novel treatment of disseminated intravascular coagulation - safety and efficacy in 1,032 patients with hematologic malignancy. Thromb Res. 2014;133(3):364-70.

55. Takezako N, Sekiguchi N, Nagata A, et al. Recombinant human thrombomodulin in the treatment of acute myeloid leukemia patients complicated by disseminated intravascular coagulation: retrospective analysis of outcomes between patients treated with heparin and recombinant human thrombomodulin therapy. Thromb Res. 2015;136(1):20-3.

56. Liu L, Yang M, Kang R, et al. HMGB1-induced autophagy promotes chemotherapy resistance in leukemia cells. Leukemia. 2011;25(1):23-31.

57. Yang J, Ikezoe T, Nishioka C, et al. Thrombomodulin-induced differentiation of acute myelomonocytic leukemia cells via JNK signaling. Leuk Res. 2012; 36(5):625-33.

58. Menell JS, Cesarman GM, Jacovina AT, et al. Annexin II and bleeding in acute promyelocytic leukemia. N Engl J Med. 1999;340(13):994-1004.

59. Matsushita T, Watanabe J, Honda G, et al. Thrombomodulin alfa treatment in patients with acute promyelocytic leukemia and disseminated intravascular coagulation: a retrospective analysis of an open-label, multicenter, post-marketing surveillance study cohort. Thromb Res. 2014; 133(5):772-81.

60. Ikezoe T, Yang J, Nishioka C, et al. Thrombomodulin enhances the antifibrinolytic and antileukemic effects of all-trans retinoic acid in acute promyelocytic leukemia cells. Exp Hematol. 2012;40(6):457-65.

61. Fujiwara $H$, Maeda $Y$, Sando $Y$, et al. Treatment of thrombotic microangiopathy after hematopoietic stem cell transplantation with recombinant human soluble thrombomodulin. Transfusion. 2016;56(4): 886-92.

62. Ohwada C, Takeuchi M, Kawaguchi T, et al. Successful treatment with recombinant soluble thrombomodulin of two cases of sinusoidal obstructive syndrome/hepatic veno-occlusive disease after bone marrow transplantation. Am J Hematol. 2011;86(10):886-8.

63. Inoue $Y$, Kosugi S, Miura I, et al. Successful treatment of refractory acute GVHD complicated by severe intestinal transplant-associated thrombotic microangiopathy using recombinant thrombomodulin. Thromb Res. 2011; 127(6):603-4.

64. Ranjan S, Goihl A, Kohli S, et al. Activated protein C protects from GvHD via PAR2/PAR3 signalling in regulatory T-cells. Nat Commun. 2017;8(1):311.

65. Rachakonda SP, Penack O, Dietrich S, et al. Single-nucleotide polymorphisms within the thrombomodulin gene (THBD) predict mortality in patients with graft-versus-host disease. J Clin Oncol. 2014;32(30):3421-7.

66. lino S, Abeyama K, Kawahara K, et al. The antimetastatic role of thrombomodulin expression in islet cell-derived tumors and its diagnostic value. Clin Cancer Res. 2004;10(18 Pt 1):6179-88.

67. Yang Y, Cheng BJ, Lu S. Thrombomodulin regulates doxorubicin sensitivity through epithelial-mesenchymal transition in non-small cell lung cancer. Eur Rev Med Pharmacol Sci. 2017;21(1):95-101.

68. Jin E, Fujiwara M, Pan X, et al. Protease-activated receptor (PAR)-1 and PAR2 participate in the cell growth of alveolar capillary endothelium in primary lung adenocarcinomas. Cancer. 2003;97(3):703-13.

69. Zheng N, Huo Z, Zhang B, et al. Thrombomodulin reduces tumorigenic and metastatic potential of lung cancer cells by up-regulation of E-cadherin and down-regulation of N-cadherin expression. Biochem Biophys Res Commun. 2016;476(4):252-9.

70. Ouchi K, Takahashi S, Chikamatsu S, et al. Retrospective analysis on the clinical outcomes of recombinant human soluble thrombomodulin for disseminated intravascular coagulation syndrome associated with solid tumors. Int J Clin Oncol. 2018;23(4):790-8.

71. Tamura K, Saito H, Asakura H, et al. Recombinant human soluble thrombomodulin (thrombomodulin alfa) to treat disseminated intravascular coagulation in solid tumors: results of a one-arm prospective trial. Int J Clin Oncol. 2015;20(4):821-8.

72. Hyman DM, Soff GA, Kampel LJ. Disseminated intravascular coagulation with excessive fibrinolysis in prostate cancer: a case series and review of the literature. Oncology. 2011;81(2):119-25.

73. Ueno H, Matsuda T, Hashimoto S, et al. Contributions of high mobility group box protein in experimental and clinical acute lung injury. Am J Respir Crit Care Med. 2004;170(12):1310-6.

74. Kudo D, Toyama M, Aoyagi T, et al. Involvement of high mobility group box 1 and the therapeutic effect of recombinant thrombomodulin in a mouse model of severe acute respiratory distress syndrome. Clin Exp Immunol. 2013;173(2):276-87.

75. Hirao S, Minakata K, Masumoto $H$, et al. Recombinant human soluble thrombomodulin prevents acute lung injury in a rat cardiopulmonary bypass model. J Thorac Cardiovasc Surg. 2017;154(6):1973-83 e1.

76. Takahashi Y, Matsutani N, Dejima H, et al. Therapeutic potential of recombinant thrombomodulin for lung injury after pneumonectomy via inhibition of high-mobility group box 1 in mice. J Trauma Acute Care Surg. 2016;81(5):868-75.

77. Kida T, Seno T, Nagahara H, et al. Roles of high-mobility group box 1 and thrombin in murine pulmonary fibrosis and the therapeutic potential of thrombomodulin. Am J Physiol Lung Cell Mol Physiol. 2018;314(3):L473-L83.

78. Oda K, Ishimoto H, Yamada S, Kushima H, Ishii H, Imanaga T, Harada T, Ishimatsu Y, Matsumoto N, Naito K, et al. Autopsy analyses in acute exacerbation of idiopathic pulmonary fibrosis. Respir Res. 2014;15:109.

79. Collard HR, Calfee CS, Wolters PJ, Song JW, Hong SB, Brady S, Ishizaka A Jones KD, King TE Jr, Matthay MA, et al. Plasma biomarker profiles in acute exacerbation of idiopathic pulmonary fibrosis. Am J Physiol Lung Cell Mol Physiol. 2010;299(1):L3-7.

80. Tsushima K, Yamaguchi K, Kono Y, Yokoyama T, Kubo K, Matsumura T, Ichimura Y, Abe M, Terada J, Tatsumi K. Thrombomodulin for acute exacerbations of idiopathic pulmonary fibrosis: a proof of concept study. Pulm Pharmacol Ther. 2014;29(2):233-40.

81. Kataoka K, Taniguchi H, Kondoh Y, et al. Recombinant human thrombomodulin in acute exacerbation of idiopathic pulmonary fibrosis. Chest. 2015;148(2):436-43.

82. Sakamoto S, Shimizu H, Isshiki T, et al. Recombinant human soluble thrombomodulin for acute exacerbation of idiopathic pulmonary fibrosis: a historically controlled study. Respir Investig. 2018;56(2):136-43.

83. Arai $\mathrm{T}$, Kida H, Ogata Y, et al. Recombinant thrombomodulin for acute exacerbation in idiopathic interstitial pneumonias. Respirology. 2019;24(7):658-66.

84. Sinibaldi S, Guzzo I, Piras R, et al. Post-transplant recurrence of atypical hemolytic uremic syndrome in a patient with thrombomodulin mutation. Pediatr Transplant. 2013;17(8):E177-81.

85. Iba T, Levy JH, Wada H, et al. Differential diagnoses for sepsis-induced disseminated intravascular coagulation: communication from the SSC of the ISTH. J Thromb Haemost. 2019;17(2):415-9.

86. Honda $T$, Ogata $S$, Mineo $E$, et al. A novel strategy for hemolytic uremic syndrome: successful treatment with thrombomodulin alpha. Pediatrics. 2013;131(3):e928-33.

87. Suyama K, Kawasaki Y, Miyazaki K, et al. The efficacy of recombinant human soluble thrombomodulin for the treatment of Shiga toxin-associated hemolytic uremic syndrome model mice. Nephrol Dial Transplant. 2015; 30(6):969-77.

88. Lattenist L, Teske G, Claessen N, et al. The lectin like domain of thrombomodulin is involved in the defence against pyelonephritis. Thromb Res. 2015;136(6):1325-31.

89. Tachibana S, Iyoda M, Matsumoto $K$, et al. Recombinant human soluble thrombomodulin attenuates anti-glomerular basement membrane glomerulonephritis in Wistar-Kyoto rats through anti-inflammatory effects. Nephrol Dial Transplant. 2019;34(5):774-82.

90. Giwa MO, Williams J, Elderfield $\mathrm{K}$, et al. Neuropathologic evidence of endothelial changes in cerebral small vessel disease. Neurology. 2012;78(3):167-74. 
91. Nakamura Y, Nakano T, Irie K, et al. Recombinant human soluble thrombomodulin ameliorates cerebral ischemic injury through a highmobility group box 1 inhibitory mechanism without hemorrhagic complications in mice. J Neurol Sci. 2016:362:278-82.

92. Su EJ, Geyer M, Wahl M, et al. The thrombomodulin analog Solulin promotes reperfusion and reduces infarct volume in a thrombotic stroke model. J Thromb Haemost. 2011;9(6):1174-82.

93. Laszik ZG, Zhou XJ, Ferrell GL, et al. Down-regulation of endothelial expression of endothelial cell protein $C$ receptor and thrombomodulin in coronary atherosclerosis. Am J Pathol. 2001;159(3):797-802.

94. Wei HJ, Li YH, Shi GY, et al. Thrombomodulin domains attenuate atherosclerosis by inhibiting thrombin-induced endothelial cell activation. Cardiovasc Res. 2011;92(2):317-27.

95. Salomaa $V$, Matei C, Aleksic N, et al. Soluble thrombomodulin as a predictor of incident coronary heart disease and symptomless carotid artery atheroscierosis in the Atherosclerosis Risk in Communities (ARIC) Study: a case-cohort study. Lancet. 1999;353(9166):1729-34.

96. Olivot J-M, Labreuche J, Aiach M, et al. Soluble thrombomodulin and brain infarction. Stroke. 2004;35(8):1946-51.

97. Hagiwara A, Tanaka N, Uemura T, et al. Can recombinant human thrombomodulin increase survival among patients with severe septicinduced disseminated intravascular coagulation: a single-centre, open-label, randomised controlled trial. BMJ Open. 2016;6(12):e012850.

98. Yamakawa K, Ogura H, Fujimi S, et al. Recombinant human soluble thrombomodulin in sepsis-induced disseminated intravascular coagulation: a multicenter propensity score analysis. Intensive Care Med. 2013;39(4):644-52.

99. Kato T, Sakai T, Kato M, et al. Recombinant human soluble thrombomodulin administration improves sepsis-induced disseminated intravascular coagulation and mortality: a retrospective cohort study. Thromb J. 2013; 11(1):3.

100. Yamato M, Minematsu Y, Fujii J, et al. Effective combination therapy of polymyxin-B direct hemoperfusion and recombinant thrombomodulin for septic shock accompanied by disseminated intravascular coagulation: a historical controlled trial. Ther Apher Dial. 2013;17(5):472-6.

\section{Publisher's Note}

Springer Nature remains neutral with regard to jurisdictional claims in published maps and institutional affiliations. 